\title{
Nucleotide sequence of the $m \times c Q$ and $m x c E$ genes, required for methanol dehydrogenase synthesis in Methylobacterium organophilum XX: a two-component regulatory system
}

\author{
H. Howard Xu, $\nmid$ Jacqueline J. Janka, Mareike Viebahn $\ddagger$ \\ and Richard S. Hanson \\ Author for correspondence: Richard S. Hanson. Tel: +1 6124717741 . Fax: +1 6124719070. \\ e-mail: hanso061@maroon.tc.umn.edu
}

Department of

Microbiology, Gray

Freshwater Biological

Institute, University of

Minnesota, PO Box 100,

Navarre, MN 55392, USA

\begin{abstract}
Nucleotide sequence analysis of the mxcQ and mxcE loci, required for the synthesis of methanol dehydrogenase in Methylobacterium organophilum XX, has revealed two open reading frames that show significant similarity to sequences of prokaryotic two-component systems, especially MxaY and MxaX proteins of another methylotrophic bacterium, Paracoccus denitrificans. Cellfree extracts and DNA-column-fractionated proteins from wild-type $\boldsymbol{M}$. organophilum XX cells grown on methanol or succinate contained protein(s) that were able to bind specifically to the upstream region of methanol dehydrogenase large subunit gene (mxaf). In contrast, cell-free extracts from mXCQ and mXcE mutant strains of $M$. organophilum XX had zero or reduced binding activity towards the promoter fragments of the mxaf gene. This is consistent with the involvement of the $m \times c Q$ and $m x c E$ genes in transcriptional regulation of methanol dehydrogenase synthesis. Analyses of sequential deletions of the mxaf upstream region have defined the functional boundary of the promoter/operator region of this gene and identified one nucleotide segment as essential to the activation of mxaF.
\end{abstract}

Keywords: methylotrophs, Metbylobacterium organopbilum XX, two-component system, methanol dehydrogenase

\section{INTRODUCTION}

Metbylobacterium organophilum XX is a pink-pigmented, facultative, type II methylotrophic bacterium (Patt et al., 1976). The enzymes involved in growth of some facultative methylotrophs on one-carbon compounds can be induced by methanol or methylamine (Anthony, 1982; O'Connor \& Hanson, 1977). The oxidation of methanol to formaldehyde is catalysed by methanol dehydrogenase $(\mathrm{MDH})$. This is a periplasmic enzyme and contains the prosthetic group pyrroloquinoline quinone (PQQ). This enzyme is abundant in all of the Gram-negative methylotrophic bacteria thus far examined (Anthony, 1982). The

\footnotetext{
†Present address: Department of Microbiology, The Ohio State University, Columbus, OH 43210-1292, USA.

¥Present address: Technische Fachhochschule Berlin, Fachbereich 3, Microbiology, Luxemburger Str. 10, 13353 Berlin, Germany.

Abbreviation: $\mathrm{MDH}$, methanol dehydrogenase.

The GenBank accession number for the sequence reported in this paper is
} U18290.
MDH enzymes of Gram-negative methylotrophs are tetramers of two types of subunits in an $\alpha_{2} \beta_{2}$ conformation (Anthony, 1982, 1986; Cox et al., 1992; Nunn et al., 1989). The large subunit ( $\alpha$ subunit) has a molecular mass of $56-76 \mathrm{kDa}$, while the small subunit $(\beta$ subunit) has a molecular mass of 8-10 kDa (Nunn et al., 1989; Cox et al., 1992; Anderson et al., 1990). The structural gene for the large subunit of $\mathrm{MDH}[m \times a F$, formerly moxF (Lidstrom et al., 1994)] from $M$. organophilum XX has been cloned and sequenced, and its promoter and transcriptional start sites have been identified (Machlin \& Hanson, 1988). Genetics studies of MDH synthesis in M. organophilum XX (Allen \& Hanson, 1985; Machlin et al., 1988; Machlin \& Hanson, 1988; Bastien et al., 1989) indicated that seven separate chromosomal regions (groups I-VII) encode a minimum of 15 genes that are required for methanol oxidation and formaldehyde assimilation. Among these genes, $m \times c Q$ and $m \times c E$ [formerly $\operatorname{moxQ}$ and $\operatorname{moxE}$ (Lidstrom et al., 1994)] have been found to be involved in the regulation of synthesis of MDH (Machlin et al., 1988; Bastien et al., 1989). 
Recently, we reported the construction of a promoterprobe vector for methylotrophic bacteria and its application in identifying methanol-regulated promoter fragments (Xu et al., 1993). This vector was also used to analyse $m \times a F$ promoter activity in vivo in $m \times c Q$ and $m \times c E$ mutant strains of $M$. organophilum and further confirmed the involvement of these two genes in transcriptional regulation of $m \times a F$ gene (Xu et al., 1993).

\section{METHODS}

Media and growth conditions. Escherichia coli JM109 was grown in Luria-Bertani medium at $37^{\circ} \mathrm{C}$. Metbylobacterium organophilum XX was grown in MacLennan minimal medium (MM) (MacLennan et al., 1971) containing 0.5\% (v/v) methanol or $0.1 \%$ succinate at $30^{\circ} \mathrm{C}$. When required, antibiotics were added at the following final concentrations: $50 \mu \mathrm{g} \mathrm{ml}^{-1}$ of ampicillin ( $\mathrm{Ap}$ ) and kanamycin $(\mathrm{Km})$ for $E$. coli strains; $15 \mu \mathrm{g} \mathrm{ml}^{-1}$ and $20 \mu \mathrm{g} \mathrm{ml}^{-1}$ of tetracycline (Tc) for E. coli and $M$. organopbilum XX, respectively.

DNA manipulation. Plasmid isolation and purification, restriction endonuclease digestion, ligation and DNA dephosphorylation were performed as described by Sambrook et al. (1989). Competent cells of E. coli JM109 were transformed with various plasmid constructs based on the protocols provided by Promega.

Cloning and sequence analysis of $m \times c Q$ and $m \times c E$ genes. A $5.5 \mathrm{~kb}$ EcoRI restriction fragment containing $m \times c Q$ and $m \times c E$ (Machlin et al., 1988) was cloned into pBluescript II SK(+) (Stratagene). From this plasmid (pBS-C6), a $2.5 \mathrm{~kb} \mathrm{BamHI}-B g l \mathrm{II}$ restriction fragment was excised, resulting in a plasmid (pBS$\mathrm{C} 6 \Delta 2.5$ ) with about $3.0 \mathrm{~kb}$ DNA insert which primarily contains $m \times c Q$ and $m \times c E$. In order to sequence this insert, five smaller fragments were further cloned into pBluescript II SK(+). Double-stranded plasmid DNA was directly used for sequencing based on protocols provided with the Sequenase Version 2.0 Sequencing Kit by US Biochemicals. Compressions were resolved by using deazanucleotide analogues in the reaction mixtures. Fourteen oligonucleotides, designed from available nucleotide sequence and synthesized by the Microchemical Facility, University of Minnesota, were used to complete the sequencing of the whole region from both DNA strands. Sequence data were analysed with the help of the University of Wisconsin Genetics Computer Group package 7.2 .

Preparation of cell-free extracts and fractionated protein solutions. Two 2-litre flasks, each containing 1 litre of MM supplemented with $0.5 \%$ methanol or $0.1 \%$ succinate, were inoculated with $5 \mathrm{ml}$ of a stationary-phase culture of an $M$. organophilum XX strain and incubated at $30^{\circ} \mathrm{C}$ with shaking (225 r.p.m.). After the culture reached $\mathrm{OD}_{600} 0 \cdot 5$, the culture was harvested by centrifugation (6000 r.p.m., $5 \mathrm{~min}$ ). The cell pellets were resuspended in $10 \mathrm{mM}$ Tris buffer $(\mathrm{pH} 7.9)$ and cell suspensions were pooled into a $30 \mathrm{ml}$ centrifuge tube. Cells were centrifuged at 6000 r.p.m. for $5 \mathrm{~min}$ and the pellet was resuspended in the Tris buffer. The washings were repeated one more time. The pellet was resuspended thoroughly in $20 \mathrm{ml}$ buffer B [12 mM HEPES/NaOH (pH 8.0), $4 \mathrm{mM}$ Tris $/ \mathrm{HCl}$ (pH 8.0), $1 \mathrm{mM}$ EDTA, $1 \mathrm{mM}$ dithiothreitol, $60 \mathrm{mM} \mathrm{KCl}$. The cell suspension was passed through a French pressure cell, twice at 15000 p.s.i. $(103 \mathrm{MPa})$, to break the cells. The broken cell suspension was collected into a glass Corex tube $(30 \mathrm{ml})$ and centrifuged for $30 \mathrm{~min}$ at $15000 \mathrm{~g}$. The cell-free supernatant was carefully collected and was ready for use. Where appropriate, the extract was directly fractionated through a DNA-cellulose
(P. L. Biochemicals) column $(0.5 \mathrm{~cm} \times 15 \mathrm{~cm})$ equilibrated with buffer $B$. The loaded column was washed with $10 \mathrm{ml}$ buffer $B$. Bound proteins were eluted sequentially with buffer $B(2 \mathrm{ml})$ containing increasing concentrations of salt $(0 \cdot 2,0 \cdot 4,0 \cdot 6,0 \cdot 8$ and $0.9 \mathrm{M} \mathrm{KCl}$ ).

Mobility-shift DNA-binding assays. These assays were performed based on the method of Chodosh (1989). Two DNA fragments containing the $m \times a F$ promoter region were used in the assay. A 353 bp DNA fragment was obtained by digesting plasmid pUS325-17 (Machlin, 1987) with AvaI and HindIII. A smaller DNA fragment (149 bp) encompassing nucleotides - 94 to +43 of the promoter region (transcription starts at +1 ) was synthesized by PCR amplification of template pUS326-17. The $5^{\prime}$ PCR primer is 5'-GAATTCGAGAAGATCGCCAA-3'. The $3^{\prime}$ PCR primer is $5^{\prime}$-AAGC'TTAGATAAACTCTGTC-3'. DNA fragments were end-labelled with $\left[\alpha^{32} \mathrm{P}\right] \mathrm{dATP}$ and used as probes. A typical binding reaction contained the following in a volume of $15 \mu \mathrm{l}: 12 \%$ glycerol, $150 \mathrm{pg}$ DNA probe (approximately 5000 c.p.m.), $4.5 \mu \mathrm{g}$ bovine serum albumin, $1 \mu \mathrm{g}$ carrier DNA [either poly $(\mathrm{dI}-\mathrm{dC}) \cdot \operatorname{poly}(\mathrm{dI}-\mathrm{dC})$, or pUC118 plasmid], $30 \mu \mathrm{g}$ or less crude protein extract or DNA-column-fractionated proteins and $20 \mathrm{ng}$ competing unlabelled DNA fragment when appropriate. The binding reaction mixture was incubated for $15 \mathrm{~min}$ at $30^{\circ} \mathrm{C}$ in a water bath. The reaction samples were loaded into wells of a $4 \%(\mathrm{w} / \mathrm{v})$ polyacrylamide gel and electrophoresed at $100 \mathrm{~V}$ for $90 \mathrm{~min}$ at $4{ }^{\circ} \mathrm{C}$ using circulating low-ionic-strength buffer $(6.7 \mathrm{mM}$ Tris/ $\mathrm{HCl}, \mathrm{pH} 7.9 ; 3.3 \mathrm{mM}$ sodium acetate; $1 \mathrm{mM}$ EDTA, pH 7.9). The polyacrylamide gel was dried on a piece of Whatman paper and the dried filter was exposed to X-ray film overnight.

Deletion mutagenesis of the mxaF promoter region. The transcriptional fusion plasmid $\mathrm{pHX} 200 \mathrm{~V}-47$ constructed previously (Xu et al., 1993), in which $x y l E$ is under control of the promoter of $m \times a F$, was used for deletional mutagenesis to identify sequences of the upstream region that are important for $m \times a F$ promoter activity and regulation. The digestion of the plasmid by restriction endonuclease, exonuclease III and S1 nuclease was performed as described by Sambrook et al. (1989). Plasmids with various lengths of deletion in the $m \times a F$ promoter region were mobilized into wild-type cells of $M$. organophilum $\mathrm{XX}$, and transconjugants grown on $\mathrm{MM}$ plus methanol were assayed for expression of the $x y l E$ gene, as described previously (Xu et al., 1993). The end-points of deletions were identified by double-stranded DNA sequencing, again as described previously (Xu et al., 1993).

\section{RESULTS}

Previous work in this laboratory has indicated that a $5.5 \mathrm{~kb}$ EcoRI restriction fragment from a group VII cosmid contains sufficient sequence to complement $m \times c Q$ and $m \times c E$ mutant strains of $M$. organopbilum XX (Machlin et al., 1988). Further subcloning complementation analysis and transposon insertional mutagenesis have localized the $m \times c Q$ and $m \times c E$ genes within a $2 \cdot 1 \mathrm{~kb} B s t$ EII and EcoRI restriction fragment (Machlin et al., 1988). Therefore, the $5.5 \mathrm{~kb}$ EcoRI restriction fragment was cloned into pBluescript II $\mathrm{SK}(+)$ and a $2.5 \mathrm{~kb} B a m \mathrm{HI}-B g l \mathrm{II}$ restriction fragment was removed from the recombinant plasmid, thus creating a plasmid with an insert size of $3.0 \mathrm{~kb}$. A $2692 \mathrm{bp}$ sequence of the insert was determined and is shown in Fig. 1.

DNA sequence analysis of this $2692 \mathrm{bp}$ region revealed two open reading frames (ORFs) which appeared to 
Two-component regulatory system in $M$. organopbilum XX

GGTGGCGCCGCTCTAGAACTAGTGGATCTGGTCACCGGACGTGACTTCACACGTCCCAAG 60 CTGCCTCAATCCGAAGACTCTGGCAGGACTCGAACACCCCCACTCGGGCGACGCGTCCCG 120 CCCOGAGCCCCCCTOGCCCGGGCAAGGTGGCCCGCCGGCGCTTCGAGAACCTCCCGGACA 180 GGGGCACGACAATAAGAGATICCTCAGTGCAGCTCGAGGCCCCAGCCGGGCTAGCAAGAT 240 GGACTITCGGCGACCAATCGGCCACGCTGCCCGGCCGACGCGAAACGCCTTCCATTCGAG 300 COGCTTAACCGTGACCGCACCCCGCACGGCCGCTTCCCGACCCGGCGATCCGECCCGCAA 360 $\max 0 Q \rightarrow$

GCGGGGCTGAGGGAGGCGGCGATGGGCCAGAGGCCGGATCACGACGCCTTCGAGGCCGCG 420 $\begin{array}{llllllllllllllll}M & G & Q & R & \text { P } & D & \text { H } & \text { D } & \text { A } & \text { F } & \text { E } & \text { A } & \text { A } & 13\end{array}$

CGCCAGGAGCGGCCCCCGGCCACCCGGGGCGCCCTGCCCTTCTGGGCCGGGCIGTCCACG 480 $\begin{array}{llllllllllllllllllllll}R & Q & E & R & P & P & A & T & R & G & A & \text { L } & \text { P } & \text { F } & \text { W } & \text { A } & G & \text { L } & \text { S } & \text { T } & 33\end{array}$

CGGATGCGGCTGATCCTGGTGGTGCTCGCCATCGACCTGGTCGCGGCCATCGCCACCTGC 540 $\begin{array}{lllllllllllllllllllll}R & M & R & L & I & L & V & V & L & A & I & D & L & V & A & A & I & A & T & C & 53\end{array}$

GGGGTCATCGTGCTCAACGCCCGACAGGCGGTGAAGGTGGAGATGCGCGCCTCCCTCGCC 600

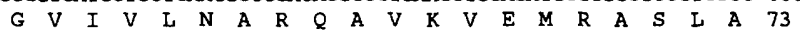

ACCGTCGAGCCCCTCGTCTCGGACACGATCCGCCTGGCCCGGGCGCCCAGCCCCGAGAGC 660 $\begin{array}{lllllllllllllllllllll}T & V & E & P & L & V & S & D & T & I & R & L & A & R & A & P & S & P & E & S & 93\end{array}$

CTGCTCCACACCCTGGATCTGCGCTTTCAGACCCTGCGCCACGTCCGCGTCGCCGTGTTC 720 $\begin{array}{lllllllllllllllllllll}\text { L } & L & H & \text { T } & \text { L } & D & \text { L } & R & \text { F } & Q & \text { T } & \text { L } & \text { R } & \text { H } & \text { V } & \text { R } & \text { V } & \text { A } & \text { V } & \text { F } & 113\end{array}$

GACGCCGCCGGCCAGCGGGTGACCATCGACGAGCCAGGGCTGCGCCGCCGCCCGCGGGAG 780 $\begin{array}{llllllllllllllllllllll}D & A & A & G & Q & R & V & T & \text { I } & \text { D } & \text { E } & \text { P } & \text { G } & \text { L } & R & R & R & P & R & E & 133\end{array}$ GCGCCGCACTGGTTOGTGCAGCTGATCGCGCCGCCGGTGGAGCAGCATCAGGTGCCGATC 840 $\begin{array}{lllllllllllllllllllll}\text { A } & P & \text { H } & \text { W } & F & V & Q & \text { L } & \text { I } & A & P & P & V & E & Q & H & Q & V & P & I & 153\end{array}$ GTGGCCGGCGGGGCGACCATCGGCACGGCCCGCATCACCACCGAGCCGGGCGACGAGATC 900 $\begin{array}{lllllllllllllllllllll}V & A & G & G & A & T & I & G & T & A & R & I & T & T & E & P & G & D & E & I & 173\end{array}$ GAGCAGAGCTGGGACGACGCCGTCTCCCTGTCGCTGGCGAGCCTGCTCCTCAACGTCGCG 960

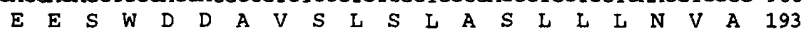
GTPCTGGCCGCGCTCTACCTCGCCTTCGGGCGGCTGCTGAAGCCGCTGCACGGCCTCGCC $102 \mathrm{C}$ $\begin{array}{lllllllllllllllllllll}V & \text { L } & \text { A } & \text { A } & \text { L } & Y & \text { L } & \text { A } & \text { F } & \text { G } & \text { R } & \text { L } & \text { L } & \text { K } & \text { P } & \text { L } & \text { H } & \text { G } & \text { L } & \text { A } & 213\end{array}$

GAAGGGCTGACCCAGCTGGAGCGGCACGACTACACCGCCCGCCTCGACCCGCCGACCTCC $108 \mathrm{C}$ $\begin{array}{llllllllllllllllllllll}E & G & L & T & Q & L & E & R & H & D & Y & T & A & R & L & D & P & P & T & S & 233\end{array}$

CGCGAGCTCGCGGTGATCACCGAGCGCTTCAACAGCGTGGCCGGGGCGTGAGCGAGGCC $114 \mathrm{C}$ $\begin{array}{llllllllllllllllllllll}R & E & L & A & V & I & T & E & R & F & N & S & V & A & G & A & L & S & E & A & 253\end{array}$

CGCTCCGCCAACAACCGCCIGAACCGGCAGCTCCTGACCGCCCAGGACGACGAGCGCCGC 1200 $\begin{array}{lllllllllllllllllllll}R & S & A & N & N & R & \text { L } & \text { N } & R & \text { Q } & \text { L } & \text { L } & \text { T } & \text { A } & \text { Q } & \text { D } & \text { D } & \text { E } & R & R & 273\end{array}$

CGCACCGCCCTGGAATTGCACGACGAGTTCGGGCCCTGCCTGTTCGCCCTCGAGGCCAAT $126 \mathrm{C}$ $\begin{array}{lllllllllllllllllllll}R & T & A & L & E & L & H & D & E & F & G & P & C & L & F & A & L & E & A & N & 293\end{array}$

GCCGCCTCCGTGGCCCGGCTCGCCCAGGCCCCGGAGCCCGACCGCGCCAAGCTCGCOGCC $132 \mathrm{C}$ $\begin{array}{lllllllllllllllllllll}\text { A } & \text { A } & \text { S } & \text { V } & \text { A } & \text { R } & \text { L } & \text { A } & \text { Q } & \text { A } & \text { P } & \text { E } & \text { P } & \text { D } & \text { R } & \text { A } & \text { K } & \text { L } & \text { A } & \text { A } & 313\end{array}$

CGGGCCGGCGACATOGCCGGCATCGTCGGGCAGGTGCAGACGCTCAACCGCGACCTGCTC $138 \mathrm{C}$ $\begin{array}{llllllllllllllllllllll}R & A & G & D & I & A & G & I & V & G & Q & V & Q & T & \text { L } & \text { N } & R & \text { D } & \text { L } & \text { L } & 333\end{array}$

AACCGCCTGCGCCCCCACGCCCTGGGCGAGGTCCCCTGCCGCAATGCCTCGACCTECTC $144 \mathrm{C}$

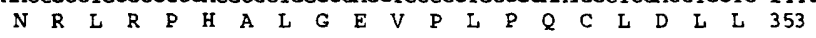

CTGCGGGATTPTTCCCGCCGGCATCCGCGCACGCGCTTCGAGGGCCATITCGAGGATCTC 1500 $\begin{array}{lllllllllllllllllllll}\text { L } & R & D & F & S & R & R & H & P & G & T & R & F & E & G & H & F & E & D & L & 373\end{array}$

GGCCGCGGCTACGGCGACGTCGTCGACCTCACCGTGTTCCGCTGCATCCAGGAGAGCATG 1560 $\begin{array}{lllllllllllllllllllll}G & R & G & Y & G & D & V & V & D & L & T & V & F & R & C & I & Q & E & S & M & 393\end{array}$

ACCAACGCCGTGCGTCACGGCGCCGCCCGCCATGTCCGCGCCGAGGCCGGCGAGACGGCG 1620 $\begin{array}{llllllllllllllllllllll}T & \text { N } & \text { A } & \text { V } & \text { R } & \text { H } & G & \text { A } & \text { A } & \text { R } & \text { H } & \text { V } & \text { R } & \text { A } & \text { E } & \text { A } & G & \text { E } & \text { T } & \text { A } & 413\end{array}$

CGGGGGCTGACGGTGAGCGTGCGGGACGACGGCAGCGGCCTCGCCCCCGACCACCGCACC 1680 $\begin{array}{lllllllllllllllllllll}R & G & L & T & V & S & V & R & D & D & G & S & G & L & A & P & D & H & R & T & 433\end{array}$ GGCCTCGGCCTCTCCGGCATGCGCGAGCGCGTGGAGGCCCTCGGCGCCAGCTTCACCCTT 1740 $\begin{array}{lllllllllllllllllllll}G & L & G & L & S & G & M & R & E & R & V & E & A & L & G & G & S & F & T & L & 453\end{array}$

GACAATGCCGCGCCGGGAACCGTTGTCCGGATCACGATCCCGATCGATCCGGAGCGGGGC 1800 $\begin{array}{lllllllllllllllllllll}\text { D } & \text { N } & \text { A } & \text { A } & \text { P } & G & \text { T } & \text { V } & \text { V } & \text { R } & \text { I } & \text { T } & \text { I } & \text { P } & \text { I } & \text { D } & \text { P } & \text { E } & \text { R } & \text { G } & 473\end{array}$

GAGGACCAGGCCGCCGGACACCCGGCCCCGGCCGCGACGCCCTCTCTCGAGACGAAGCCC 1860

$\begin{array}{llllllllllllllllllllll}E & D & Q & A & A & G & H & P & A & P & A & A & T & P & S & L & E & T & K & P & 493\end{array}$

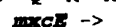

GCGACATGACTGCGCTTGCGATGAAGACCCCCGCCGACTCCCTCGCCAAGGGCACCCCCG 1920 A $T$ *

$\begin{array}{lllllllllllllllllll}M & \text { T } & \text { A } & L & \text { A } & \text { M } & \text { K } & \text { T } & \text { P } & \text { A } & \text { D } & S & L & \text { A } & K & G & T & P & 18\end{array}$
CCGAGACCGTGCTCGTGATCGACGATCATCCGATCGTCCTCCAGGGCTGCCGCCGCGTGC 1980 $\begin{array}{llllllllllllllllllllll}\text { A } & \text { E } & \text { T } & \text { V } & \text { L } & \text { V } & \text { I } & \text { D } & \text { D } & \text { H } & \text { P } & \text { I } & \text { V } & \text { L } & \text { Q } & \text { G } & \text { C } & \text { R } & \text { R } & \text { V } & 38\end{array}$

TGGAGGATGCCGGCATCGAGAGCGTGGCCGAGGCGACCGGCACGGTCGCCGGCTACCGCC 2040 $\begin{array}{lllllllllllllllllllll}\text { L } & \text { E } & \text { D } & \text { A } & \text { G } & \text { I } & \text { E } & \text { S } & \text { V } & \text { A } & \text { E } & \text { A } & \text { T } & \text { G } & \text { T } & \text { V } & \text { A } & \text { G } & \text { Y } & \text { R } & 58\end{array}$

TFTTCCACCGCCTGCGCCCGAAGGTOGTGATCTGCGACCTCACCTTCCAGGGCAGCGGAT 2100 $\begin{array}{llllllllllllllllllllll}\text { L } & F & H & R & \text { L } & R & \text { P } & K & \text { V } & \text { V } & \text { I } & \text { C } & \text { D } & \text { L } & \text { T } & \text { F } & \text { Q } & \text { G } & \text { S } & G & 78\end{array}$

TGGCCGGCCTCGCCCTGATCCGCCGCATGCGCGCCGTGGAGCCGGGCACGCGGGTGCTCG 2160 $\begin{array}{lllllllllllllllllllll}\text { L } & A & G & \text { L } & \text { A } & \text { L } & \text { I } & R & R & M & R & A & V & E & \text { P } & G & T & R & V & L & 98\end{array}$

TGTTCTCGATGCACAACGACCCGGTCATCGTCTCGCGCGCCCTCGAAGCCGCGGCGCTCG 2220 $\begin{array}{llllllllllllllllllllll}\text { V } & \text { F } & \text { S } & \text { M } & \text { H } & \text { N } & \text { D } & \text { P } & \text { V } & \text { I } & \text { V } & \text { S } & \text { R } & \text { A } & \text { L } & \text { E } & \text { A } & \text { G } & \text { A } & \text { L } & 118\end{array}$

GCTACGTGCTCAAGCACCACGCCTCCAACGAGCTGTTCCAGGCCTICGAGCAGGTGCGCA 2280 $\begin{array}{lllllllllllllllllllll}G & Y & \text { V } & \text { L } & \text { K } & \text { D } & \text { H } & \text { A } & \text { S } & \text { N } & \text { E } & \text { L } & \text { F } & \text { Q } & \text { A } & \text { F } & \text { E } & \text { Q } & \text { V } & \text { R } & 138\end{array}$

AGGGCGAGGTCTTCCTCGACCGCCGGCTCGCCACCGAGGTGGCGATGCTGCGGGCCGACC 2340 $\begin{array}{llllllllllllllllllllll}\text { K } & G & \text { E } & \text { V } & \text { F } & \text { L } & \text { D } & \text { R } & \text { R } & \text { L } & \text { A } & \text { T } & \text { E } & \text { V } & \text { A } & \text { M } & \text { L } & \text { R } & \text { A } & \text { D } & 158\end{array}$

CGCGCCGCACCCCCGAATCCCAGCTCACCCCCCGCGAGCAGCAGATCCTGGCCCGCCTCG 2400

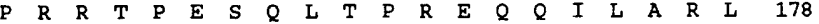

CCCAGGGCAAGTCCTACGGCGCGATCGCGGCCGACCTCTCGGTCAGCTACAAGACCGTCA 2460 $\begin{array}{llllllllllllllllllllll}A & \& & G & K & S & Y & G & A & I & A & A & D & L & S & V & S & Y & K & T & V & 198\end{array}$

CCAATGCCTGCTCGCAGATGCGCCAGAAACTCGGCGTGCGCGCCCTGGCCGAGCTGATCC 2520 $\begin{array}{lllllllllllllllllllll}T & N & A & C & S & Q & M & R & Q & K & \text { L } & G & \text { V } & \text { R } & \text { A } & \text { L } & \text { A } & \text { E } & \text { L } & \text { I } & 218\end{array}$

ACGTGGCCGTGACCCAGGCGCAGGGCAGGGGIGAGACGTCGCCTCCGGTTGCGTAGAGGG 2580

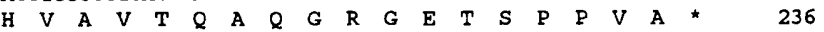

GGTCGAGCGCCTITCCTTCCCCTCTGGCGAAGGTGGCCGCGAAGACGGACGAGGCGGCAC 2640

CGAAGTACCOGGCATCGAGCGCGGCGCGGCCTCCACCGCGCTCTCCCCCTCG

2692

Fig. 1. Nucleotide sequence of the 2692 bp DNA fragment and deduced amino acid sequences of the $m \times c Q$ and $m \times c E$ genes from $M$. organophilum XX. Putative ribosome-binding sites are underlined. Asterisks indicate the ends of the protein sequences.

correspond to the proposed $m \times c Q$ and $m \times c E$ positions and sizes (Fig. 1) (Machlin et al., 1988). Both ORFs have putative ribosome-binding sites characteristic of $M$. organophilum $\mathrm{XX}$ genes. The $m \times<Q$ gene starts at nt 382 and ends at nt 1869 , and would encode a 495 amino acid polypeptide with an estimated molecular mass of $54 \mathrm{kDa}$. The $m \times c E$ gene starts at nt 1866 and ends at 2576, encoding a polypeptide of 236 amino acids with an estimated molecular mass of $26 \mathrm{kDa}$. Gene bank searches revealed that $m \times c Q$ and $m \times c E$ belong to the histidine kinase and response regulator families, respectively.

\section{MxcQ is a member of the histidine kinase superfamily}

The C-terminal deduced amino acid sequence of $m \times c Q$ was found to be similar to MxaY [formerly MoxY (Lidstrom et al., 1994)] of Paracoccus denitrificans (Harms et al., 1993), and a number of other histidine kinases from this superfamily (Fig. 2). MxcQ and MxaY have the highest sequence similarity over entire amino acid sequences ( $29 \%$ identity over 420 amino acid overlap) (Fig. 2). As expected for a histidine kinase, MxcQ contained several residues that are especially conserved in its C-terminal portion. A conserved histidine precedes a conserved asparagine by about 100 residues (Stock $e t$ al., 1989). A stretch of 15-45 amino acid residues separates this asparagine and a five-residue region designated DXGXG (where $X$ residue is not conserved). Twenty to 50 residues away from DXGXG, there follows a GXG 


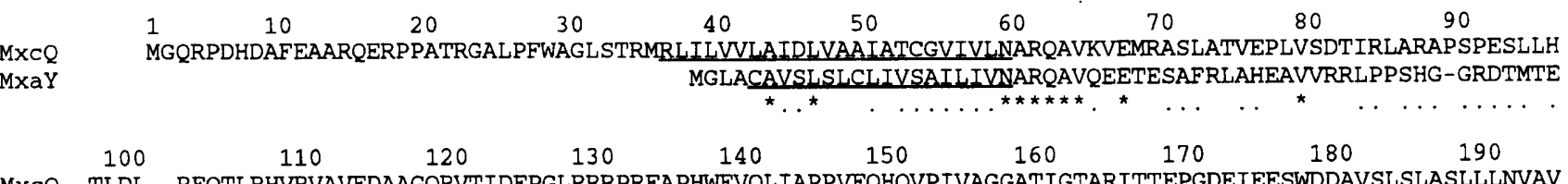

MXCO TLDL--RFOTLRHVRVAVFDAAGQRVTIDEPGLRRRPREAPHWFVQLIAPPVEQHQVPIVAGGATIGTARITTEPGDEIEESWDDAVSLSLASLLLNVAV

MXaY AIGLAEEIDGLRHVSARILDPEGQPLQHRAHGQLRSEASAPQWFSALMTPPLVEALVPITHYPNVLGMLRVAADPTDEIAEVWGD-FSIILPVLFLAGLA

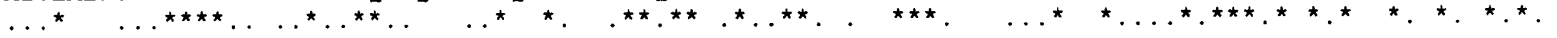

$\begin{array}{lllllllll}200 & 210 & 220 & 230 & 240 & 250 & 260 & 270 & 280\end{array}$

MXCQ LAAL-YLAFGRLLKPLHGLAEGLTQLERHDYTARLDPPTSRELAVITERFNSVAGALSEARSANNRLNRQLLTAQDDERRRTALELHDEFGPCLFALEAN MXaY MVGLAFLMTTLLTRRLOSVOAAMAMODGRLSVRAPDDRLTEFADLAAGVNALASHLQAEQAENDLLQARLIGSSEAERSRIALDLHDEMGPQLFALRA-

UhpB

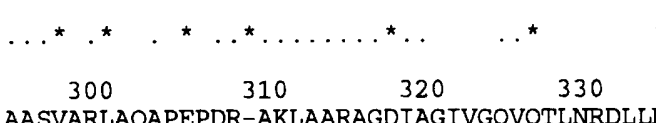
TEFADLAAGVNALASHLQAEQAENDLLQARLIGSSEAERSRIALDLHDEMGPQLFALRA-
-LGRGLYFNQAQKHFQQLLLME----ERATIARELHDSLAQVLSYLRIQ
-LTATLALDRHQERQQQLIVME----ERATIARELHDSIAQSLSCMKMQ
-VGLLLADAQAKQD-FGLRIIEAQEEERKRVSREIHDGPAQMLANVMMR $*$ *... $* \underset{\star}{- \text { LNQSLQKELARNHRLAERLLETEESVRRDVARELHDDIC }}$ $340 \quad 350 \quad 360 \quad 370 \quad 380$

MXCQ AASVARLAQAPEPDR-AKLAARAGDIAGIVGQVQTLNRDLLNRLRPHALGEVPLPQCLDLLLRDF SRRHPGTR--FEGHF -EDLGRGYGDVVDLTVFRCI MXaY AVSHAOAMTADLPERPAALDETLDAIAGHALEVORSARTAINDLRPMLLGEASLAELLAELVTGFRDVASETRVVLDVDP-EVEGSSPGELAELSIYRFA NarO LTLLKR--SIPEDN--ATAOSIMADF SOALNDAYROLRELLTTFR-LTLOOADLPSALREMLDTLQ-NQTSAKLTLDCRLPTLA---LDAQMOVHLLQI I NarX VSCLQM--QGDALP--ESSRELLSQIRNELNASWAQLRELLTTFR-LQLTEPGLRPALEASCEEYS-AKFGFPVKLDYQLPPRL---VPSHQAIHLLQIA DegS SELIERIFRDRGAE--DGFQEIKNLRQNVRNALYE-VRRIIYDLRPMALDDLGLIPTLRKYLYTTEEYNGKVKIHFQ-CIGETEDQRLAPQFEVALFRLA UhPB AGIVQRLAADNG----GVKQSGQLIEHVSLGVYDAVRRLLGRLRPRQLDDLTLAQAIRSLVREMELESRGIVSHLDWRIDETA---LSESQRVTLFRVC

390 400 410 420 430 440 450 MXCO OESMTIAVRHGAARHVRAFAG- - ETARGLTVSVRDDGSGIAPD-_. MXaY RESVLNAMRHGRATVVRVSLDTMPDEPGQIVVRVTDNGRGPQSGTG-_... NarQ REAVLNAMKHANASEIAVSC--VTAPDGNHTVYIRDNGIGIGEPKEPEGH---YGLNIMRERAERLGGTLTFSQPSGGGTLVSISFRSAEGEESQLM NarX REALSNALKHSOASEVVVT--.VAONDNOVKLTVODNGCGVPENAIRSNH---YGMIIMRDRAOSLRGDCRVRRRESGGTEVVVTF IPEKTFTDVQGDTH DegS OEAVSNALKHSESEEITVK---VEITKDFVILMIKDNOKGFDLKEAKEKKNKSFGLLGMKERVDLLEGTMTIDSKIGLCTFIMIKVPLSL

UhPB QEGLNNIVKHANASAVTLQ---GWQQDERLMLVIEDDGSGL-

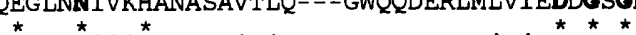

$$
\begin{array}{llll}
460 & 470 & 480 & 490
\end{array}
$$

MXCQ PGTVVRITIPIDPERGEDQAAGHPAPAATPSLETKPAT

MXIY NRLTHTELRMPRPCKLR

NarX E

UhPB GTRVSVSLPQRYV

Fig. 2. Amino acid sequence alignment of MxcQ with the entire MxaY sequence of $P$. denitrificans (Harms et al., 1993) and C-terminal parts of NarQ and NarX of E. coli (Nohno et al., 1989; Rabin \& Stewart, 1992), DegS of Bacillus subtilis (Tanaka \& Kawata, 1988; Henner et al., 1988), and UhpB of Salmonella typhimurium (Island et al., 1992). Numbers indicate MxcQ amino acid positions. Identical residues at a given position are marked by an asterisk at the bottom of the alignment. Residues of the same conserved amino acid groups are indicated by a dot. Putative transmembrane domains are underlined. Residues in bold face are highly conserved among histidine kinases.

conserved block. In the $\mathrm{MxcQ}$ protein sequence, $\mathrm{H}-280$ is separated by 115 amino acids from conserved N-395, followed by a 27 -residue spacing, and the conserved $D$ 422XG-424XG-426. Interestingly, the GXG conserved regions in MxcQ (Fig. 2) and MxaY (Harms et al., 1993) are only 7 and 10 residues, respectively, away from the conserved DXGXG regions, a much shorter distance than in the majority of the histidine kinase family (Fig. 2) (Stock et al., 1989). Hydrophobicity analysis of MxcQ protein has located two potential transmembrane helices (positions 36-59 and 182-204 in Fig. 2), aligned similarly to the putative transmembrane helices of $\mathrm{MxaY}$ protein (Harms et al., 1993).

\section{MxcE is a member of the response regulator family}

The response regulator family is defined by a conserved domain of about 100 amino acids that are generally located near the $\mathrm{N}$-terminus of these proteins (Stock $e t$ al., 1989). Based on sequence similarities between their Cterminal domains, these response regulators may be placed into several subfamilies (Stock et al., 1989). The deduced amino acid sequence of $m \times c E$ was found to belong to a response regulator subfamily including $\mathrm{MxaX}$ [formerly MoxX (Lidstrom et al., 1994)] of $P$. denitrificans (Harms et al., 1993) (Fig. 3). MxcE has the highest sequence similarity to MxaX of $P$. denitrificans ( $35 \%$ identity in 217 amino acids overlap) (Fig. 3). As can be seen in Fig. 3, there are three amino acid residues universally conserved in the N-terminal domains of these response regulators: an aspartic acid residue corresponding to D-27 of $\mathrm{MxcE}$, another aspartic acid residue corresponding to D-71 of $\mathrm{MxcE}$, and a lysine residue corresponding to position $\mathrm{K}$ 123. The second aspartic acid position has been found to be the site of phosphorylation by histidine kinases of the two-component systems (Stock et al., 1989; Hess et al., 1988; Igo et al., 1989). The C-terminal sequence of $\mathrm{MxcE}$ was also found to align very well with the $\mathrm{C}$-terminal domains of other proteins in this subfamily (Fig. 3), with the following characteristic patterns (Kahn \& Ditta, 1991): (i) one block of similarity around the conserved Arg/Glu pair at R-170/E-171 of MxcE protein; (ii) a 


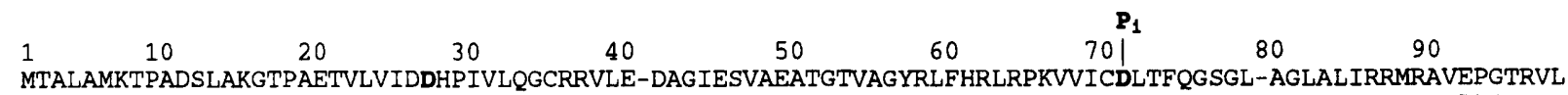
$\begin{array}{ll}\text { MxCE } & \text { MTALAMKTPADSLAKGTPAETVLVIDDHPIVLQGCRRVLE-DAGIESVAEATGTVAGYRLFHRLRPKVVICDLTFQGSGL-AGLALIRRMRAVEPGTRVL } \\ \text { MxaX } & \text { MOA-ALKLOSDD-ARPL---OILIVDDHPVVAEGWGRIIR-TKTACEIASAPSASEGWRAWRQARPDLMVVDLSIGRNKI-AGIRLIERLRRVDPDLPIL }\end{array}$ DegU MT---_..---KVNIVIIDDHOLFREGVKRILDFEPTFEVVAEGDDGDEAARIVEHYHPDVVIMDINMP--NV-NGVEATKOLVELYPESKVI NarL MS--------NQEPATILLIDDHPMLRTGVKQLISMAPDITVVGEASNGEQG IELAESLDPDLILLDLNMP--GM-NGLETLDKLREKSLSGRIV UhpA M--.-------ITVALIDDHLIVRSGFAQLLGLEPDLQVVAEFGSGREALAGL PGRGVQVCICDISMP--DI-SGLELLSQLPK----GMATI BVgA MY---NKV--.-_-..---LIIDDHPVLRFAVRVLMEKE-GFEVIGETDNGIDGLKIAREKIPNLVVLDIGIP--KL-DGLEVIARLQSLGLPLRVL RCSB MN---.-----NMNVI IADDHPIVLFGIRKSLEQIEWVNVVGEFEDSTAL INNLPKLDAHVLITDLSMPGDKYGDGITLIKYIKRHFPSLSII FixJ M--...--PESLPVHVIDDDDAVRESLAFLLESSGL--AVTQHTSAAAFLDAGVPLDRGCIVTDVRMPG---ISGLELLKELNARGAHMAVI $\ldots \star \ldots$

100 110

120

130

140

150

160

170

180

MXCE VFSMHNDPVIVSRALEAGALGYVLXDHASNELFQAFEOVRKGEVFLDRRLATEVAM-_._._. MXaX VFTMHRSPVLARRALMFGANGIINRDSPPAEICAAFTEVARGGNYVDSRLAMOIAL_............. LNVSRPGTSAPRLTGREEEILGMITECM DegU ILSIHDDENYVTHALKTGARGYLLKEMDADTLIEAVKVVAEGGSYLHPKVTHNLVNEFRRLATSGVSAHPQHEVYPEIRRPLHILTRRECEVLQMLADGK NarL VFSVSNHEEDVVTALKRGADGYLLKDMEPEDLLKALHQAAAGEMVLSEALTPVLAASLR-----ANRATTERDVNQ-------LTPRERDILKLIAQGL

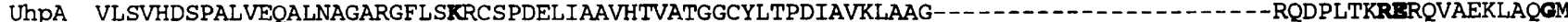
BVgA VLTGQPPSLFARRCLNSGAAGFVCKHENLHEVINAAKAVMAGYTYF PSTTLSEMRMG-_......-.-DNAKSDSTLISVLSNRBLTVLQLLAQGM

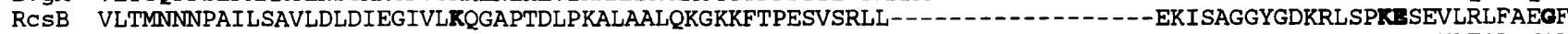

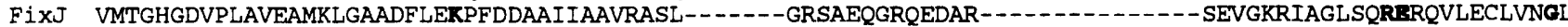
190
200
210
220
230

法

MXaX SYRDIADRACISYKTVSNVSLVLKDKLGAANLADLVVKGIRYFEGD

DegU SNRGIGESLFISEKTVKNHVSNILQKMNVNDRTQAVVVAIKNGWVEMR

NarL PNKMIARRLDITESTVKVHVKHMLKKMKLKSRVEA-AVWVHQERIF

UhPA AVKEIAAELGLSPKTVHVHRANLLEKLGVSNDVE-LAHRMFDGW

BVGA SNKDIADSMFLSNKTVSTYKTRLLQKLNATSLVELIDLAKRNNLA

RCSB LVTEIAKKLNRSIKTISSQKKSAMMKLGVENDIALLNYLSSVTLSPADKD

FIXJ ANKTIAYDLGISPRTVEVYRANVMTRMKAASLPELVRMALLAGVAPADDATPT

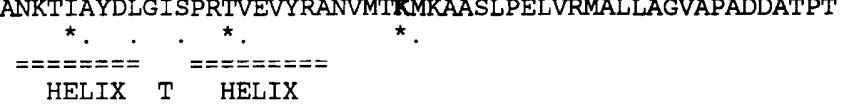

Fig. 3. Amino acid sequence alignment of MxcE with MxaX of P. denitrificans (Harms et al., 1993), DegU of $B$. subtilis (Tanaka \& Kawata, 1988; Henner et al., 1988), NarL of E. coli (Nohno et al., 1989), UhpA of S. typhimurium (Island et al., 1992), BvgA of Bordetella pertussis (Arico et al., 1989), RcsB of E. coli (Stout \& Gottesman, 1990) and FixJ of Azorhizobium caulinodans (Kaminski \& Elmerich, 1991). The site of phosphorylation is marked 'Pi'. Numbers indicate MxcE amino acid positions. Identical residues at a given position are marked by an asterisk at the bottom of the alignment. Residues of the same conserved amino acid groups are indicated by a dot. Residues in bold face are highly conserved among this subfamily of response regulators.

(a)

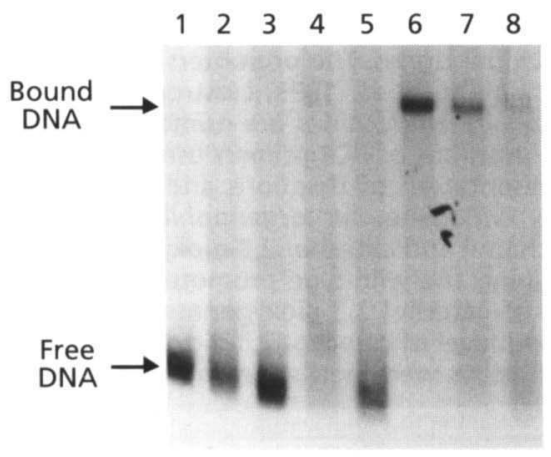

(b)

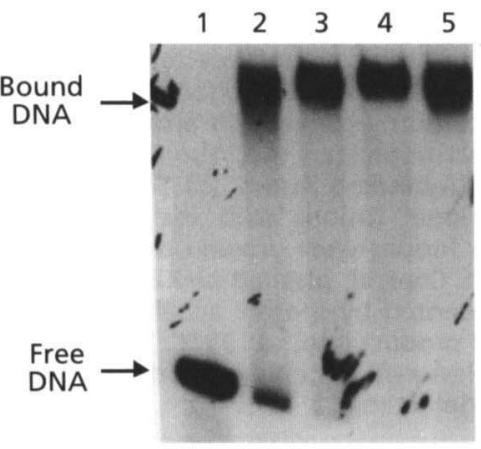

(c)

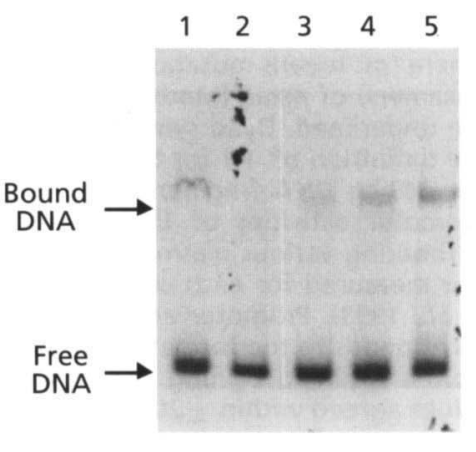

Fig. 4. Mobility-shift DNA-binding assays. (a) DNA-binding assay using a 353 bp Aval and Hindll DNA fragment (150 pg DNA probe per well). Crude protein extract (lane 8) as well as DNA-column-fractionated proteins (eluted at $0.6 \mathrm{M} \mathrm{KCl}$; named $D$ fraction) (lanes 2-7) were added to the reaction mixture. These extracts were from $M$. organophilum XX grown in MM plus methanol. Lane 1, DNA probe control; lanes 2 and 3, $1.7 \mathrm{ng} \mathrm{D}$ fraction; lanes 4 and 5, 3.4 ng D fraction; lanes 6 and $7,6.8 \mathrm{ng} D$ fraction; lane $8,10 \mu \mathrm{g}$ crude protein extract; reactions in lanes 3,5 and 7 each contained $20 \mathrm{ng}$ unlabelled competitive DNA fragment. (b) DNA-binding assay using a 149 bp PCR-amplified DNA fragment (140 pg DNA probe per well). Lane 1, DNA probe control; lanes 2,3 and 4 contain $6.8 \mathrm{ng}, 13.6 \mathrm{ng}$ and $27 \mathrm{ng}$, respectively, of D fraction; lane $5,38 \mu \mathrm{g}$ crude protein extract. The extracts were the same as above. (c) DNA-binding assay of mutant cellular extracts using a 149 bp PCR-amplified fragment (140 pg DNA probe per well). Lane 1, 149 bp DNA probe control; lane 2, $25 \mu \mathrm{g}$ of crude protein extract from mutant strain SM4 (an mxcQ mutant) grown with succinate; lane 3, $22 \mu \mathrm{g}$ of crude protein extract from mutant strain SM49 (an mxcE mutant) grown with succinate; lane 4, $26 \mu \mathrm{g}$ crude protein extract from wild-type strain grown with succinate; lane 5, 6.8 $\mathrm{ng} \mathrm{D}$ fraction from the wild-type strain grown with methanol. 
(a)

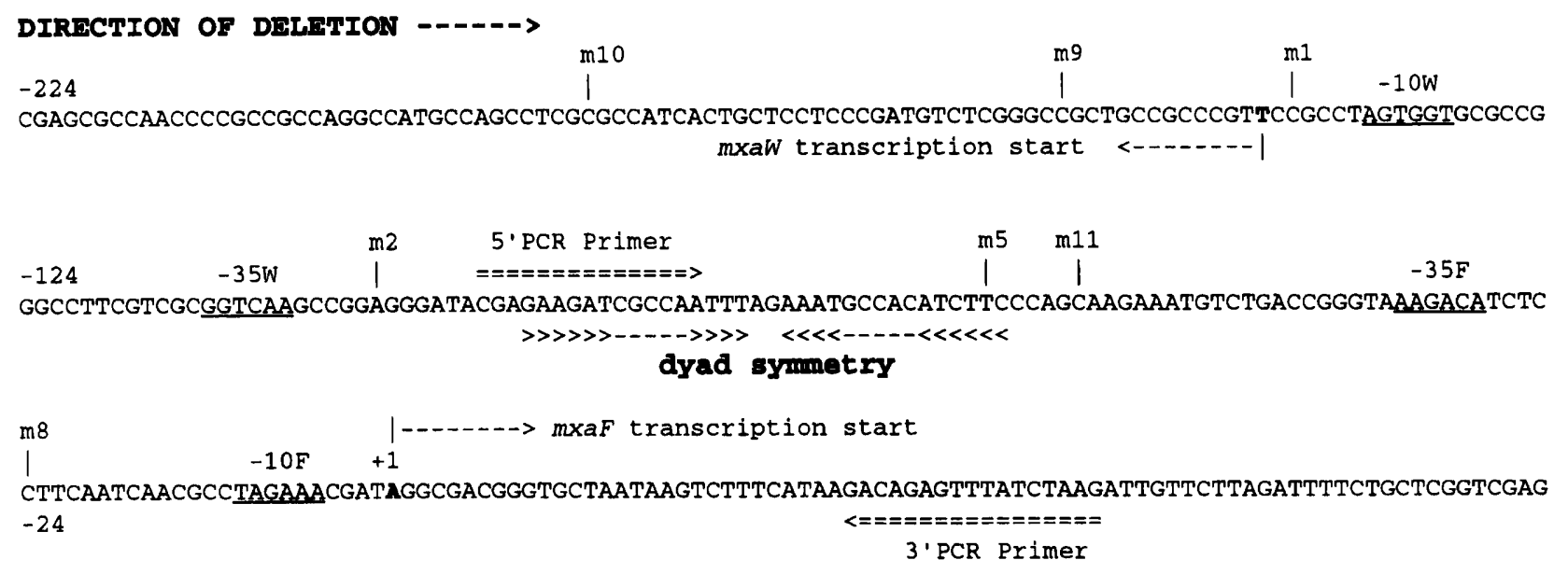

(b) Promoter activity

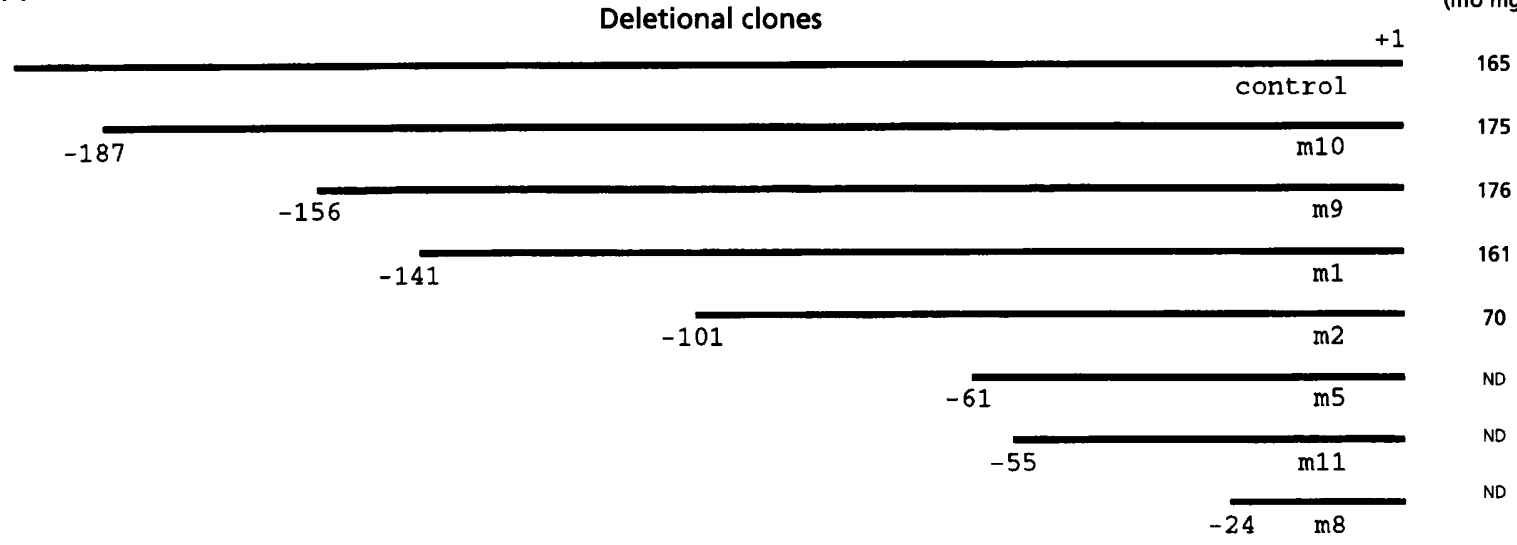

Fig. 5. Deletional mutagenesis of the $m x a F$ promoter fragment. (a) Nucleotide sequence of the $m \times a F$ promoter region (presented previously by Machlin \& Hanson, 1988, and Xu et al., 1993). Deletion end-points are individually marked 'mN', where ' $m$ ' means mutation and ' $N$ ' is the Arabic numeral designated for individual clones. The promoters (-35 and - 10 hexamers) of mxaF (Machlin \& Hanson, 1988) and mxaW genes [formerly moxW (Xu et al., 1993; Lidstrom et al., 1994)] are underlined. Dyad symmetry is indicated by converging arrows $(>>><<<$ ). Nucleotides are numbered based on the definition of +1 for the mxaF transcription start site. Also shown are the positions of PCR primers used to synthesize the 149 bp DNA fragment used for DNA-binding assays. (b) Schematic representation of deletions and corresponding promoter activities of their transcriptional fusions with the promoterless $x y l E$ gene. $M$. organophilum $X X$ strains harbouring various plasmids containing fusions were grown on MM plus methanol and catechol 2,3-dioxygenase activity was measured for each cell-free extract. Control, plasmid pHX200V-47 containing the wild-type promoter fragment (Xu et al., 1993). Promoter activity is represented by specific activity (mU $\mathrm{mg}^{-1}$ ) of catechol 2,3-dioxygenase. One milliunit corresponds to the formation of $1 \mathrm{nmol}$ product $\mathrm{min}^{-1}$ at $30^{\circ} \mathrm{C}$. The background level of catechol 2,3 -dioxygenase activity from a strain containing the promoterless $x y l E$ gene was not detectable. All assays were performed at least twice, and values agreed within $\pm 20 \%$. ND, None detectable.

conserved Gly at position 181 ; (iii) a putative helix-turnhelix motif between positions 184 and 203; and (iv) another block of similarity around the highly conserved lysine at position 208.

\section{Extracts of $m \times c Q$ and $m \times c E$ mutants show no or reduced binding to promoter fragments of the $m \times a F$ gene}

Nucleotide sequences and transcriptional start sites of mxaF genes from $M$. organopbilum XX and Metbylo- bacterium extorquens AM1 have been identified (Machlin \& Hanson, 1988; Anderson et al., 1990). Not only are the two ORFs of the $m \times a F$ genes well conserved, but the two genes have identical -35 and -10 promoter hexamers as well as Shine-Dalgarno sequences (Machlin \& Hanson, 1988; Anderson et al., 1990). Due to the fact that $m \times a F$ was shown to be induced by methanol, an obvious point of regulation would be at the level of transcription. Mobility-shift DNA-binding assays (Fig. 4) were performed using a $353 \mathrm{bp} A v a \mathrm{I} /$ HindIII restriction fragment and a PCR-amplified $149 \mathrm{bp}$ DNA fragment containing 
the $m x a F$ promoter region from $M$. organophilum $\mathrm{XX}$ (the positions of the PCR primers are shown in Fig. 5a). A DNA band shift was observed when a crude extract or the protein fraction eluted by $0.6 \mathrm{M} \mathrm{KCl} /$ buffer $B$ from the DNA-cellulose column was incubated with the promoter fragments (Fig. 4a, b). DNA-column fractionation was shown to greatly concentrate DNA-binding activity (data not shown). The retarded band decreased in intensity when an excess of competitive (unlabelled) DNA fragment was added, indicating the existence of DNA-binding protein(s) capable of binding specifically to the promoter region (Fig. $4 a$ ). On the other hand, the $m \times c Q$ mutant of $M$. organophilum $\mathrm{XX}$ grown in succinate produced no proteins that bound specifically to the $m x a F$ promoter region (Fig. 4c), even though wild-type cells grown with succinate also produce protein(s) that bind to the promoter fragments (Fig. 4c). Succinate-grown cells have MDH with approximately $25 \%$ of the specific activity of methanol-grown cells (data not shown). A DNA band shift with reduced density was observed with the cell-free extract from the $m \times c E$ mutant (Fig. 4c).

\section{Definition of the functional boundary of the mxaF upstream regulatory region}

The difference in DNA-binding activities between extracts of $M$. organophilum XX wild-type strain and mutant strains implies a regulatory role for the $m \times a F$ upstream region. Therefore, deletions of the $m \times a F$ upstream region were constructed to confirm the importance of $m \times a F$ upstream sequence to regulation. Sequential deletions were made from the distal end of the $m \times a F$ upstream region on plasmid pHX200V-47 (Xu et al., 1993) and the promoter activities of the mutant promoter regions were examined in $M$. organophilum XX based on the expression of the reporter gene $x y l E$. As shown in Fig. 5(a, b), the first three deletions (m10, $\mathrm{m}$ ) and $m 1$ ) from the distal (left) end had no apparent effects on $m x a F$ promoter activity $(x y l E$ expression was almost the same as in the strain harbouring control plasmid pHX200V-47). This indicated that the deleted nucleotides (between -324 and -140 based on the transcription start at +1 ) were not important in regulating $x y l E$ expression. Deletion $\mathrm{m} 2$ caused a $59 \%$ reduction in promoter activity, although the -35 hexamer of the promoter is still $76 \mathrm{bp}$ away. The next three deletions ( $\mathrm{m} 5, \mathrm{~m} 11$ and $\mathrm{m} 8$ ) completely abolished the promoter activity. These results indicated that nucleotides between -103 and -62 were essential for $m \times a F$ promoter activity. The sequence immediately upstream of -103 may be involved in the regulation of $m \times a F$, but it is not essential.

\section{DISCUSSION}

In this report, we have described the nucleotide sequence of the $m \times c Q$ and $m \times c E$ genes required for the synthesis of methanol dehydrogenase in $M$. organophilum $\mathrm{XX}$. Through database searches, the deduced amino acid sequences of $\mathrm{MxcQ}$ and $\mathrm{MxcE}$ polypeptides were shown to show similarity with the histidine kinase and response regulator families, respectively. Especially significant is the finding that $\mathrm{MxcQ}$ and $\mathrm{MxcE}$ proteins have the highest similarity with another pair of two-component regulators, $\mathrm{MxaY}$ and $\mathrm{MxaX}$, respectively, of the methylotrophic bacterium $P$. denitrificans (Figs 2 and 3; Harms et al., 1993). The amino acid residues that are conserved among histidine kinases and response regulators (Stock et al., 1989) are also found in the MxcQ and $\mathrm{MxcE}$ proteins (Figs 2 and 3). These observations imply that the $m \times c Q$ and $m \times c E$ genes of $M$. organophilum $\mathrm{XX}$ are equivalent to the $m \times a Y$ and $m \times a X$ genes of $P$. denitrificans, although their locations relative to the regulated genes $(m \times a F)$ are different. $m \times a Y$ and $m \times a X$ are linked to $m \times a F$ on the chromosome of $P$. denitrificans (Harms et al., 1998), while $m \times c Q$ and $m \times c E$ are located at least $40 \mathrm{~kb}$ away from the gene cluster containing $m \times a F$ (Machlin et al., 1988). As for $m \times a Y$ and $m \times a X$, there is no intergenic sequence between the $m \times c Q$ and $m \times c E$ coding sequences, implying co-transcription of these two genes (Fig. 1).

Mutant strains of $M$. organophilum XX with defects in $m \times c Q$ and $m \times c E$ have previously been isolated (Machlin $e t$ al., 1988). These mutants (formerly designated VII-A, VII-B mutants) cannot grow on methanol, but retain the ability to grow on methylamine and succinate (Machlin $e t$ al., 1988). During growth on methylamine, $m \times c Q$ and $m \times c E$ mutants do not synthesize proteins that cross-react with anti-MDH antisera (Machlin et al., 1988). Furthermore, no reporter gene transcription was detected in these mutants when they harboured plasmids containing $m \times a F p:: x y l E$ transcriptional fusions (Xu et al., 1993). The discovery in this study that $m \times c Q$ and $m \times c E$ genes probably encode a histidine kinase and a response regulator, respectively, not only reaffirms the conclusion that $m \times c Q$ and $m \times c E$ genes are required for the synthesis of MDH (Machlin et al., 1988; Xu et al., 1993; Lidstrom et al., 1994) but also indicates the possible mechanism of regulation involved.

It is reasonable to propose that, like other pairs of twocomponent systems (Nohno et al., 1989; Stock et al., 1989), $m \times c Q$ and $m \times c E$ are constitutively produced by $M$. organophilum XX cells. After sensing the signal (whose identity is not yet known), MxcQ protein may autophosphorylate itself, thus activating its kinase activity, presumably located at its C-terminus. Then MxcQ phosphorylates the $\mathrm{MxcE}$ protein present in the cytoplasm. Activated $\mathrm{MxcE}$ protein may bind to the promoter region of the $m \times a F$ gene (possibly other genes), activating its transcription. DNA-binding results indicated that the $m \times c Q$ mutant does not produce protein(s) that bind to the $m \times a F$ promoter region, consistent with the role of a histidine kinase. Less clear is the result of DNA binding by extracts of the $m \times c E$ mutant; the cell-free extract still contained DNA-binding activity, although at a reduced level. This can be explained by the possibility of a mutation in $\mathrm{MxcE}$ protein that renders it incapable of activating $m \times a F$ transcription but does not prevent its binding to the target site upstream of $m \times a F$. Alternatively, $\mathrm{MxcE}$ may activate the transcription of yet another regulatory protein which directly activates the $m \times a F$ gene. The presence of a putative helix-turn-helix domain 
in the MxcE protein supports its function as a DNAbinding protein, just like most other response regulators (Stock et al., 1989).

In vivo analyses of deletion mutant plasmids have indicated that nucleotides upstream of -140 (relative to the transcription start of $m x a F$ ) had no effect on the function of $m x a F$ promoter fused to the $x y l E$ gene and nucleotides between -140 and -103 only slightly affected the activity of the $m \times a F$ promoter. In contrast, nucleotides between -103 and -62 were absolutely essential for the expression of $x y / E$, implying a positive regulatory mechanism for control of the $m x a F$ promoter. Interestingly, a dyad symmetry was found to span nucleotides -91 and -60 (Fig. 5a), providing a potential binding site for a positive regulatory factor.

de Vries et al. (1990) proposed that a GC-rich consensus sequence appearing upstream of several $P$. denitrificans genes is responsible for regulating gene expression in that bacterium. Support for this hypothesis was found in $M$. organophilum XX because a sequence with partial similarity to this consensus was located more than 300 bp upstream of the transcriptional start site of $m \times a F$ from strain $\mathrm{XX}$ (de Vries et al., 1990). However, the results of deletion analysis clearly indicated that nucleotides upstream of position -140 are not required for normal transcription and regulation of $m \times a F$ in $M$. organophilum $\mathrm{XX}$.

\section{ACKNOWLEDGEMENTS}

This work was funded by a grant from the US Department of Energy (DOE-F602-88ER 13682). We would like to thank anonymous reviewers for their comments to improve the manuscript.

\section{REFERENCES}

Allen, L. N. \& Hanson, R. S. (1985). Construction of broad-hostrange cosmid cloning vectors: identification of genes necessary for growth of Metbylobacterium organophilum XX on methanol.J Bacteriol 161, 955-962.

Anderson, D. J., Morris, C. J., Nunn, D. N., Anthony, C. \& Lidstrom, M. E. (1990). Nucleotide sequence of the Metbylobacterium extorquens AM1 moxF and moxJ genes involved in methanol oxidation. Gene 90, 173-176.

Anthony, C. (1982). The Biochemistry of Methylotrophs. New York: Academic Press.

Anthony, C. (1986). Bacterial oxidation of methane and methanol. Adv Microb Pbysiol 27, 113-210.

Arico, B., Miller, J., Roy, C., Stibitz, S., Monack, D., Falkow, S., Gross, R. \& Rappuoli, R. (1989). Sequences required for expression of Bordetella pertussis virulence factors share homology with prokaryotic signal transduction proteins. Proc Natl Acad Sci USA 86, 6671-6675.

Bastien, C., Machlin, S., Zhang, Y., Donaldson, K. \& Hanson, R. S. (1989). Organization of genes required for the oxidation of methanol to formaldehyde in three type II methylotrophs. Appl Environ Microbiol 55, 3124-3130.

Chodosh, L. A. (1989). Mobility shift DNA-binding assay using gel electrophoresis. In Current Protocols in Molecular Biology, pp. 12.2.112.2.10. Edited by F. M. Ausubel, R. Brent, R. E. Kingston, D. D.
Moore, J. G. Seidman, J. A. Smith \& K. Struhl. New York: John Wiley.

Cox, J. M., Day, D. J. \& Anthony, C. (1992). The interaction of methanol dehydrogenase and its electron acceptor, cytochrome $c_{\mathrm{L}}$, in methylotrophic bacteria. Biocbim Biophys Acta 1119, 97-106.

Harms, N., Reijnders, W. N. M., Anazawa, H., van der Palen, C. J. N. M., van Spanning, R. J. M., Oltmann, L. F. \& Stouthamer, A. H. (1993). Identification of a two-component regulatory system controlling methanol dehydrogenase synthesis in Paracoccus denitrificans. Mol Microbiol 8, 457-470.

Henner, D. J., Yang, M. \& Ferrari, E. (1988). Localization of Bacillus subtilis $\operatorname{sac} \mathrm{U}(\mathrm{Hy})$ mutations to two linked genes with similarities to the conserved procaryotic family of two-component signaling systems. J Bacteriol 170, 5102-5109.

Hess, J., Oosawa, K., Kaplan, N. \& Simon, M. I. (1988). Phosphorylation of three proteins in the signaling pathway of bacterial chemotaxis. Cell 53, 79-87.

Igo, M. M., Ninfa, A. J. \& Silhavy, T. J. (1989). A bacterial environmental sensor that functions as a protein kinase and stimulates transcriptional activation. Genes \& Dev 3, 598-605.

Island, M. D., Wei, B.-Y. \& Kadner, R. J. (1992). Structure and function of the $u b p$ genes for the sugar phosphate transport system in Escherichia coli and Salmonella typhimurium. J Bacteriol 174, 2754-2762.

Kahn, D. \& Ditta, G. (1991). Modular structure of Fix J : homology of the transcriptional activator domain with the -35 binding domain of sigma factors. Mol Microbiol 5, 987-997.

Kaminski, P. A. \& Elmerich, C. (1991). Involvement of fixLJ in the regulation of nitrogen fixation in Azorbizobium caulinodans. Mol Microbiol 5, 665-673.

Lidstrom, M. E., Anthony, C., Biville, F., Gasser, F., Goodwin, P., Hanson, R. S. \& Harms, N. (1994). New unified nomenclature for genes involved in the oxidation of methanol in Gram-negative bacteria. FEMS Microbiol Lett 117, 103-106.

Machlin, S. M. (1987). Genetic studies of methanol debydrogenase synthesis in the facultative methylotroph Methylobacterium organophilum $X X . \mathrm{PhD}$ thesis, University of Minnesota.

Machlin, S. M. \& Hanson, R. S. (1988). Nucleotide sequence and transcriptional start site of the Metbylobacterium organophilum XX methanol dehydrogenase structural gene. J Bacteriol 170, 4739-4747.

Machlin, S. M., Tam, P. E., Bastien, C. A. \& Hanson, R. S. (1988). Genetic and physical analyses of Methylobacterium organophilum XX genes encoding methanol oxidation. $J$ Bacteriol 170, 141-148.

MacLennan, D. G., Onsby, J. C., Vasey, R. B. \& Cotton, N. T. (1971). The influence of dissolved oxygen on Pseudomonas AM1 grown on methanol in continuous culture. $J$ Gen Microbiol 69, 395-404.

Nohno, T., Noji, S., Taniguchi, S. \& Saito, T. (1989). The nar $X$ and nar $L$ genes encoding the nitrate-sensing regulators of Escherichia coli are homologous to a family of prokaryotic two-component regulatory genes. Nucleic Acids Res 17, 2947-2957.

Nunn, D. N., Day, D. \& Anthony, C. (1989). The second subunit of methanol dehydrogenase of Methylobacterium extorquens AM1. Biochem J 260, 857-862.

O'Connor, M. L. \& Hanson, R. S. (1977). Enzyme regulation in Methylobacterium organophilum. J Gen Microbiol 98, 265-272.

Patt, T. E., Cole, G. C. \& Hanson, R. S. (1976). Methylobacterium, a new genus of facultative methylotrophic bacteria. Int J Syst Bacteriol 26, 226-229.

Rabin, R. S. \& Stewart, V. (1992). Either of two functionally redundant sensor proteins, $\mathrm{NarX}$ and $\mathrm{NarQ}$, is sufficient for nitrate 
regulation in Escherichia coli K-12. Proc Natl Acad Sci USA 89, 8419-8423.

Sambrook, J., Fritsch, E. F. \& Maniatis, T. (1989). Molecular Cloning: a Laboratory Manual. Cold Spring Harbor, NY: Cold Spring Harbor Laboratory.

Stock, J. B., Ninfa, A. J. \& Stock, A. M. (1989). Protein phosphorylation and regulation of adaptive responses in bacteria. Microbiol Rev 53, 450-490.

Stout, V. \& Gottesman, S. (1990). RcsB and RcsC: a twocomponent regulator of capsule synthesis in Eschericbia coli. $J$ Bacteriol 172, 659-669.
Tanaka, T. \& Kawata, M. (1988). Cloning and characterization of Bacillus subtilis iep, which has positive and negative effects on production of extracellular proteases. J Bacteriol 170, 3593-3600.

de Vries, G. E., Kues, U. \& Stahl, U. (1990). Physiology and genetics of methylotrophic bacteria. FEMS Microbiol Rev 75, 57-102.

Xu, H. H., Viebahn, M. \& Hanson, R. S. (1993). Identification of methanol regulated promoter sequences from the facultative methylotrophic bacterium Methylobacterium organophilum XX. J Gen Microbiol 139, 743-752.

Received 15 February 1995; revised 16 May 1995; accepted 7 June 1995. 